\title{
Assessment of Special Endurance of Athletes in Freestyle Wrestling at the Preparatory Stage of a Sports Cycle
}

\author{
Sargylana S. Kuzmina ${ }^{*}$; Marianna I. Solovieva ${ }^{1}$; V. R. Abramova ${ }^{2}$; N. N Sivtsev ${ }^{2}$; \\ E. V. Korkin ${ }^{2}$, Kristina S. Gavrileva ${ }^{3}$; Natalya V. Makharova ${ }^{3}$ \\ ${ }^{1}$ M. K. Ammosov North-Eastern Federal University, \\ ${ }^{2}$ The Churapcha State Institute of Physical Education and Sports \\ ${ }^{3}$ School of the Highest Sports Skill \\ Yakutsk, the Sakha Republic, Russia
}

\begin{abstract}
We evaluated the special endurance (SE) of freestyle wrestlers with the help of complex tests of special working capacity, while simultaneously monitoring the change in levels of lactate concentration and LPO in the blood at the preparatory stage of the annual sport cycle. A total of 23 athletes with high sports qualification participated in the experiment. Athletes were tested in the field by "repeated marginal load" tests, including the operational test (OT) developed by the authors. The most noticeable shifts in the studied indicators were observed after the test load of OT, which indicates an increase in the glycolytic capacity of the athlete's body. Thus, our study shows the possibility of using our OT to analyze the anaerobic performance of elite athletes in freestyle wrestling. (International Journal of Biomedicine. 2017;7(3):251-253.)
\end{abstract}

Key Words: freestyle wrestling $\bullet$ special endurance $\bullet$ lactate $\bullet$ lipid peroxidation

\section{Abbreviations}

EI, endurance index; LPO, lipid peroxidation; LMAO, low molecular weight antioxidants; MDA, malondialdehyde; OT, operational test; SE, special endurance.

\section{Introduction}

At present, in the theory of sports training of athletes in freestyle wrestling, the growth of sports achievements is associated with an increase in the technical preparedness of athletes. The Churapcha State Institute of Physical Education and Sports (ChSIPES), actively conducts research to optimize the technical training of athletes engaged in freestyle wrestling. The trainers of ChSIPES, summarizing the long-term experience, identify the priority means of technical training of athletes and construct the programs, taking into account the specifics and traditions of the institute. Improving the technical and tactical training of athletes in freestyle wrestling requires the development of SE, given the increased intensity

*Corresponding author: Sargylana S. Kuzmina. North-East Federal University, Yakutsk, the Republic of Sakha (Yakutia), Russia. E-mail: sskuzmina@bk.ru of competitive activity. In accordance with the specifics of the wrestling match, the increase of SE is due to an accelerated involvement of anaerobic glycolytic processes in the energy supply of the work. It is possible to assess the level of development of the SE of athletes in freestyle wrestling by the indicator of the magnitude of the acidotic shifts in a situation as close as possible to a competitive one (1) At the same time, the intensity of anaerobic glycolytic processes reflects the concentration of lactate in the blood. The analysis of data in the literature indicates that the most suitable conditions for physical exercise, in which the maximum realization of the anaerobic glycolytic potential occurs, is an intermittent load with a repeated short-term series of the maximum possible load lasting no more than 60 seconds for each exercise, with constant (1-3 minute or shorter) rest intervals. ${ }^{(2)}$ With intensive physical load, a limiting factor, along with an increase in lactate concentration, is the accumulation of LPO products. In accordance with V. Lankin et al., ${ }^{(3)}$ moderate activation 
of LPO processes in response to the adverse factor is one of the adaptation mechanisms. However, the accumulation of products of free radical oxidation is the most important disadaptation factor; it reduces the motor abilities of the organism, its endurance and physical working capacity.

The topic of this study is relevant because coaches and athletes need a selection of simple and effective medicobiological methods for assessing the SE of athletes throughout the training process.

The purpose of our work was to assess the SE of freestyle wrestlers with the help of complex tests of special working capacity while monitoring the level of changes in lactate concentration and the LPO level in the blood at the pre-competition stage of the annual training cycle.

\section{Material and Methods}

The study involved 23 students of ChSIPES with the sports qualifications of the candidates for Master of Sports and Master of Sports aged between 18 and 23, who are engaged in freestyle wrestling. The study protocol was reviewed and approved by the Ethics Committee of the North-Eastern Federal University. All participants provided the written informed consent.

To determine the concentration of lactate in the peripheral blood, a portable device, Accutrend Lactate (Roche Diagnostics), was used, which allows one to determine the level of lactate in a training hall for 1 minute. The blood test was performed 3 minutes after the exercise. The LPO intensity was determined according to the serum MDA level. MDA content in serum was determined by spectrophotometric method by reaction with thiobarbituric acid at $\lambda=532 \mathrm{~nm}$. The content LMAO was determined by by ortho-phenanthroline colour method. ${ }^{(4)}$ o-Phenantroline quantitatively forms complex with $\mathrm{Fe}^{2+}$, which get disrupted in the presence of chelating agents. The antioxidant interfered with the formation of ferrousophenantroline complex which is spectrophotometrically read at $510 \mathrm{~nm}$.

Blood for examination was taken from the ulnar vein in the morning before and at the end of training sessions. To assess $\mathrm{SE}$, a complex test was performed according to VF Boyko, ${ }^{(5)}$ in which, in the interval mode, the athlete performs 3 specific loads of a different nature with maximum available intensity during 20 seconds with intervals of rest of 10 seconds. The EI was calculated as the ratio of the average values between data obtained during the second and third minute of work and data recorded during the first minute. The second type of test was OT developed by the ChSIPES trainers for the rapid assessment of special technical preparedness. This is a set of special exercises performed three times: the 10 throws through the back with capturing a hand ("poker"), taking into account the performance time; the 10 sit-ups on one leg, taking into account performance time; the 10 jumps imitating the protection action against the passage to the feet ("throwing off the legs"), taking into account performance time; the 10 throws through the hip, taking into account performance time; the 10 torso lifts, lying on the back, hands behind the head ("torso lifts"), taking into account performance time. A conditional unit (CU), the ratio of the average data of the first approach to the average data of the second and third approaches, was also calculated.

Results were statistically processed using the software package BIOSTAT 3.03 and Microsoft Excel 2007. The mean (M) and standard error of the mean (SEM) were calculated. Student's paired t-test was used to compare the differences between the paired samples. A probability value of $\mathrm{P}<0.05$ was considered statistically significant.

\section{Results and Discussion}

Based on the results of pedagogical testing of the level of special technical preparedness of wrestlers at the beginning and at the end of training camps, a reliable increase in the parameters of the complex test was revealed in 5 students out of 23; the remaining students did not show any significant differences in the studied parameters. There was also no significant shift in lactate concentration after the test. According to EI in the complex test, a slight increase in this value was observed. It is possible that in the performance of this test, the athletes were not motivated to perform the limit work, and the results obtained cannot show the degree to which they realized their functional capabilities.

In the OT, the students showed improved results (Table 1). According to throws through the back with capturing a hand, the gain was 15 seconds, on average, "torso lifts" - 18 seconds, "throwing off the legs" - 10 seconds. In general in the OT, the coefficient increased by $20 \%(P<0.05)$. The average lactate value after the test also increased by $11 \%(P<0.05)$, which may indicate an increase in the anaerobic glycolytic capacity of the bodies of athletes. An increase in the blood lactate level after the test, with improved test results, indicates a predominantly glycolytic anaerobic orientation of this test load.

Table 1.

Results of tests of special working capacity

\begin{tabular}{|l|c|c|c|c|}
\hline \multirow{2}{*}{ Test } & \multicolumn{2}{|c|}{$\begin{array}{c}\text { At the beginning of } \\
\text { training cycle }\end{array}$} & \multicolumn{2}{c|}{$\begin{array}{c}\text { At the end of } \\
\text { training cycle }\end{array}$} \\
\cline { 2 - 5 } & $\begin{array}{c}\text { Test } \\
\text { Score }\end{array}$ & $\begin{array}{l}\text { lactate, } \\
\text { mmol/1 }\end{array}$ & $\begin{array}{c}\text { Test } \\
\text { Score }\end{array}$ & $\begin{array}{c}\text { lactate, } \\
\text { mmol/1 }\end{array}$ \\
\hline Complex test ${ }^{(5)}$ EI & $0.68 \pm 0.06$ & $11.8 \pm 1.1$ & $0.71 \pm 0.09 *$ & $12.5 \pm 1.2 *$ \\
\hline OT, CU & $0.72 \pm 0.05$ & $16.5 \pm 1.3$ & $0.89 \pm 0.08^{*}$ & $18.5 \pm 2.6^{*}$ \\
\hline
\end{tabular}

$*_{-} P<0.05$ - compared with the beginning of the training cycle

From the literature, it is known that in people adapted to physical exertion, the level of LPO is lowered and the amount of antioxidants in muscles is increased. In athletes adapted to speed-power loads, an increase in the intensity of LPO is found. ${ }^{(6)}$ Earlier, we found an increase in the LPO level and a decrease in the degree of antioxidant protection in the blood of athletes compared with the control group. ${ }^{(7)}$ To determine the impact of LPO processes on the performance of athletes, we determined the MDA content and the total activity of LMAO 
in the blood at the beginning and at the end of this stage of the training cycle. The results showed a significant increase in the blood concentration of MDA and LMAO by $10 \%$ and $6 \%$, respectively, indicating an increase in the intensity of the "LPO-antioxidants" system, which may have a negative effect on the general and special performance of wrestlers.

\section{Conclusion}

The results of assessing the SE of freestyle wrestlers in two tests give reason to assume that the OT mainly reflects the anaerobic performance of athletes. Improving the results according to data of this test and deeper acidotic changes in the testing of athletes indicate an increase in the glycolytic capacity and SE of athletes at this stage of the training cycle. The increased tension in the "LPO-antioxidants" system in the body of wrestlers requires antioxidant correction of LPO activation caused by intense physical exertion. It is beneficial to test the special physical working capacity of wrestlers with biochemical control of lactate concentration changes at different stages of training in order to assess the dynamics of the development of SE and to manage the training process.

\section{Competing interests}

The authors declare that they have no competing interests.

\section{References}

1. Shiyan VV, Igumenova LA. [Assessment of bioenergetic potential for wrestlers]. M.: SportAkadem Press, 2003. [in Russian].

2. KarpmanVL, Belotserkovsky ZB, Gudkov IA. [Studies of physical performance in athletes]. Moscow: FiS; 1974. [in Russian].

3. Lankin VZ, Tikhaze AK, Belenkov YuN. [Free radical processes in normal and pathological conditions]. M.: NIIK; 2001. [in Russian].

4. Rogozhin VV, Kuryliuk TT. [Methods of biochemical analysis]. Yakutsk: YaGU; 1997. [in Russian].

5. Boyko VF. Structure and diagnostics of special endurance of qualified wrestlers. Abstract of PhD Thesis. Kiev;1982. [in Russian].

6. Ignat'ev AV, Chernikova AG, Khmel'kov AV. Features of the ratio of processes of lipid peroxidation and the level of antioxidant activity in the body of individuals adapted to various types of physical activity. In: Koshsheev VS, editor. Medico-technical problems of individual protection of a person: Individual protection of a person in activities in extreme conditions. M.: Institute of Biophysics;1987. [in Russian].

7. Gavrileva KS, Khandi MV, Solovieva MI, Kuzmina SS. [Features of the functional state and antioxidant system in young athletes engaged in mass wrestling]. Vestnik Sportivnoi Nauki. 2016;(1):29-33. [Article in Russian]. 